\title{
Associations of estimated $\Delta-5$-desaturase and $\Delta-6$-desaturase activities with stroke risk factors and risk of stroke: the Kuopio Ischaemic Heart Disease Risk Factor Study
}

\author{
Roya Daneshmand, Sudhir Kurl, Tomi-Pekka Tuomainen and Jyrki K. Virtanen* \\ Kuopio Campus, Institute of Public Health and Clinical Nutrition, University of Eastern Finland, PO Box 1627, \\ 70211 Kuopio, Finland
}

(Submitted 28 September 2016 - Final revision received 30 January 2017 - Accepted 14 February 2017)

\section{Abstract}

Stroke is a leading cause of morbidity and mortality. The role of PUFA in reducing the risk of stroke is uncertain. The concentrations of PUFA in the human body are determined both by dietary intake and by activities of desaturase enzymes. Desaturase enzymes have been associated with chronic diseases, but little is known about their association with stroke risk. We investigated the associations of $\Delta$-6-desaturase (D6D) and $\Delta$-5-desaturase (D5D) activities with stroke risk factors and risk of stroke among 1842 men from the prospective, population-based Kuopio Ischaemic Heart Disease Risk Factor Study, aged 42-60 years and free of CVD at baseline in 1984-1989. ANCOVA and Cox regression models were used for the analyses. Whole serum desaturase activities were estimated as product:precursor ratios $-\gamma$-linolenic acid:linoleic acid for D6D and arachidonic acid:dihomo- $\gamma$-linolenic acid for D5D. Higher D6D activity was associated with higher systolic and diastolic blood pressure, BMI, serum insulin and TAG concentrations and worse homoeostatic model assessment (HOMA) indices. In contrast, higher D5D activity was associated with lower systolic and diastolic blood pressure, BMI, serum insulin, LDL-cholesterol, TAG and C-reactive protein concentrations, higher HDL-cholesterol concentration, and better HOMA indices. During the mean follow-up of 21.2 years, 202 stroke cases occurred. Neither D6D activity (multivariable-adjusted extreme-quartile hazard ratios (HR) 1.18; 95\% CI 0.80, 1.74) nor D5D activity (HR 1.06; $95 \%$ CI $0.70,1.60)$ were associated with stroke risk. In conclusion, higher D5D activity was favourably associated and higher D6D activity unfavourably associated with several stroke risk factors, but not with the risk of incident stroke.

\section{Key words: Desaturases: PUFA: Stroke risk factors: Risk factors: Prospective studies}

PUFA have an important role in the prevention of CVD ${ }^{(1,2)}$. Levels of the essential fatty acids linoleic acid (LA) and $\alpha$-linolenic acid in the body are determined by diet, but the levels of other PUFA are influenced not only by diet, but also by desaturase enzymes $^{(3)} \cdot \Delta-5$ desaturase (D5D) and $\Delta-6$ desaturase (D6D) catalyse the endogenous synthesis of long-chain PUFA from the essential fatty acids and are considered key enzymes for PUFA conversion $^{(4,5)}$. Especially D6D is a rate-limiting enzyme in the whole PUFA pathway, because it is the enzyme that converts the $n-6$ PUFA LA and the $n-3$ PUFA $\alpha$-linolenic acid to longerchain $n-6$ and $n-3$ PUFA. D5D and D6D are encoded, respectively, by the fatty acid desaturase (FADS) genes FADS1 and $F A D S 2$, which have been shown to be the strongest genetic predictors of circulating PUFA concentrations ${ }^{(6)}$.

D5D and D6D activities and FADS1 and FADS2 polymorphisms have been associated with risk of chronic diseases, such as type 2 diabetes and $\mathrm{CVD}^{(3)}$. However, little is known about the association with stroke risk $^{(7)}$, although $\mathrm{D} 5 \mathrm{D}$ and $\mathrm{D} 6 \mathrm{D}$ activities have been rather consistently associated with several stroke risk factors, such as high blood pressure, high BMI and obesity, worse serum lipid profile, inflammation and insulin resistance. In general, D6D activity has been associated with a higher risk of these factors ${ }^{(8-14)}$ and D5D activity with a lower risk $^{(8-13,15-17)}$. However, many of the studies have been small. Therefore, in order to elucidate the role of $\mathrm{D} 5 \mathrm{D}$ and $\mathrm{D} 6 \mathrm{D}$ activities in the development of stroke, we investigated the associations of the estimated D5D and D6D activities with risk of incident stroke and cross-sectionally with stroke risk factors in 1842 middle-aged and older men from Finland.

\section{Methods}

\section{Study design and population}

The Kuopio Ischaemic Heart Disease Risk Factor Study (KIHD) was designed to explore the associations between risk factors and

Abbreviations: D5D, $\Delta-5$ desaturase; D6D, $\Delta-6$ desaturase; FADS, fatty acid desaturase; HOMA, homoeostatic model assessment; ICD, International Classification of Diseases; LA, linoleic acid.

* Corresponding author: J. K. Virtanen, fax +35817162 936, email jyrki.virtanen@uef.fi 
risk of CVD, atherosclerosis, stroke and other chronic diseases ${ }^{(18)}$. The baseline examinations were performed in 1984-1989. All men who were $42,48,54$ or 60 years old and living in the city of Kuopio or surrounding areas were invited and $2682(82.9 \%$ of those eligible) participated in the baseline examinations. This study was conducted according to the guidelines laid down in the Declaration of Helsinki and all procedures involving human subjects were approved by the Research Ethics Committee of the University of Kuopio. Written informed consent was obtained from all participants. Men with a history of CVD or stroke $(n$ 709) or with missing data on serum PUFA ( $n$ 131) were excluded, leaving 1842 men.

\section{Serum fatty acid measurements}

Serum esterified fatty acids and NEFA were specified in one GC run without preseparation as described ${ }^{(19)}$. Serum fatty acids were extracted with chloroform-methanol. The chloroform phase was evaporated and treated with sodium methoxide, which methylated esterified fatty acids. Quantification was carried out with reference standards purchased from NU-Chek Prep Inc. Each analyte had an individual reference standard, and the internal standard was eicosane. Fatty acids were chromatographed in an NB-351 capillary column (HNU-Nordion) using a Hewlett-Packard 5890 Series II GC (Hewlett-Packard Company, Agilent Technologies Inc. since 1999) with a flame ionisation detector. Results were obtained in $\mathrm{mmol} / \mathrm{l}$ and presented as proportion of total serum fatty acids. CV for repeated measurements of fatty acids was $9 \cdot 6 \%$ for LA $(18: 2 n-6), 11.7 \%$ for $\gamma$-linolenic acid (18:3n-6), 8.3\% for dihomo- $\gamma$-linolenic acid $(20: 3 n-6)$ and $9 \cdot 2 \%$ for arachidonic acid (20:4n-6). Desaturase enzyme activities were estimated as the ratio of product: precursor and were calculated as the ratio of arachidonic acid: dihomo- $\gamma$-linolenic acid for D5D activity and as the ratio of $\gamma$-linolenic acid:LA for D6D activity ${ }^{(20)}$.

\section{Other measurements}

The subjects gave fasting blood samples between 08.00 and 10.00 hours at the baseline examinations in 1984-1989. They were instructed to abstain from ingesting alcohol for $3 \mathrm{~d}$ and from smoking and eating for $12 \mathrm{~h}$ before giving the sample. Detailed descriptions of the determination of serum lipids and lipoproteins, assessment of medical history and medications, family history of diseases, smoking and alcohol consumption, have been published ${ }^{(21)}$. Plasma glucose was measured using a glucose dehydrogenase method after precipitation of proteins by TCA. Serum insulin was determined with a Novo Biolabs Radioimmunoassay Kit (Novo Nordisk). Diabetes was defined as self-reported diabetes mellitus or fasting blood glucose of $6.7 \mathrm{mmol} / \mathrm{l}$ or more. Insulin resistance and sensitivity and $\beta$-cell function were estimated by the homoeostatic model assessment (HOMA) computer algorithm ${ }^{(22,23)}$. Education was assessed in years by using a self-administrated questionnaire. Physical activity was assessed using the KIHD 12-Month Leisure-Time Physical Activity Questionnaire ${ }^{(24)}$. Serum C-reactive protein (CRP) was measured with an immunometric assay (Immulite High Sensitivity CRP Assay; DPC). BMI was computed as the ratio of weight $(\mathrm{kg})$ :the square of height $\left(\mathrm{m}^{2}\right)$. Dietary intake of foods and nutrients was assessed at the time of blood sampling using a 4-d food recording ${ }^{(25)}$.

\section{Ascertainment of follow-up events}

Incident strokes between the years 1984 and 1992 were observed through the FINMONICA stroke register ${ }^{(26)}$. Information regarding stroke incidents between years 1993 and 2012 was collected through computerised linkage to the national hospital discharge registry. The diagnosis of stroke was based on sudden onset of clinical signs or focal or global disturbance of cerebral function lasting $24 \mathrm{~h}$ (except in the case of sudden death or if interrupted by surgical intervention) with no apparent cause other than a vascular origin. Each suspected stroke (International Classification of Diseases (ICD)-9 codes 430-439 and ICD-10 codes I60-I68 and G45-G46) was classified into: (1) a definite stroke, (2) no stroke or (3) an unclassifiable event. The FINMONICA stroke register data were annually rechecked with the data obtained from the computerised national hospital discharge and death registers. Definite strokes and unclassifiable events were included in the group of any stroke. Each definite stroke was classified into: (1) an ischaemic stroke (ICD-9 codes 433, 434; ICD-10 code I63) or (2) a haemorrhagic stroke (ICD-9 codes 430, 431; ICD-10 codes I60-I61). If the subject had multiple non-fatal strokes during follow-up, the first stroke was considered as the end point. Computed tomography (CT) was performed in $90 \%$ of the patients by 1993 , and CT, MRI and autopsy reached $100 \%$ by $1997^{(27)}$. Every resident of Finland has a unique personal identifier that is used in registers. There were no losses to follow-up.

\section{Statistical analysis}

Subjects were divided into quartiles according to estimated D6D and D5D activities. The univariate relationships between estimated D6D and D5D activities and baseline characteristics were assessed by means and linear regression (for continuous variables) or $\chi^{2}$ tests (for categorical variables). The mean values of risk factors in the estimated D6D and D5D activity quartiles were analysed using ANCOVA. Associations between estimated D6D and D5D activities and risk of incident stroke were analysed using Cox regression models. Two different models were used to adjust for potential confounders. The first model was adjusted for age and examination year. The second model further included BMI, smoking, physical activity and alcohol intake. All quantitative variables were entered as continuous variables. The covariates in the models were chosen to comply with recent analyses regarding the $n-3$ and $n-6$ PUFA and risk of stroke in this study population ${ }^{(28)}$. Further adjustment for the potential confounders education and waist circumference did not appreciably change the associations (change in estimates $<5 \%$ ). Cohort mean was used to replace missing values in covariates $(<0.5 \%)$. Tests of linear trend were conducted by assigning the median values for each category of exposure variable and treating those as a single continuous variable. All $P$ values were two-tailed $(\alpha$ 0.05). Data were analysed using SPSS 23.0 for Windows (IBM Corp.). 


\section{Results}

At baseline, higher estimated D5D activity was mainly associated with lower BMI and higher alcohol intake, whereas higher estimated D6D activity was associated with lower age, lower education, higher BMI and higher alcohol intake (Table 1). They were also more likely to live in a rural area and have type 2 diabetes. Those with higher estimated D5D activity had higher serum concentrations of total $n-3$ PUFA, total $n-6$ PUFA, LA and arachidonic acid but lower $\gamma$-linolenic acid and dihomo- $\gamma$-linolenic acid concentrations, whereas those with higher estimated D6D activity had higher concentrations of EPA, DPA, $\gamma$-linolenic acid, dihomo- $\gamma$-linolenic acid and arachidonic acid but lower concentrations of total $n-6$ PUFA, total $n$-3 PUFA, LA and DHA (Table 1 ).

Table 2 shows the associations of the estimated desaturase activities with stroke risk factors. After multivariable adjustments, higher estimated D6D activity was associated with higher diastolic blood pressure, BMI, serum insulin concentration, serum TAG concentration, HOMA insulin resistance and HOMA $\beta$-cell function, and lower HOMA insulin sensitivity. Although the $P_{\text {trend }}$ across the quartiles was statistically significant for the association with systolic blood pressure as well, visual inspection did not support a linear association (Table 2). In contrast, higher estimated D5D activity was associated with lower systolic and diastolic blood pressure, BMI, serum insulin concentration, serum LDL-cholesterol concentration, serum TAG concentration, CRP concentration, HOMA insulin resistance and HOMA $\beta$-cell function, and higher HDL-cholesterol concentration and HOMA insulin sensitivity. No statistically significant associations were found with blood glucose.

During the average follow-up of 21.2 years (min-max 0.3-28.8 years), 202 men (11.0\%) experienced a stroke. Of all strokes, 153 were ischaemic strokes and fifty-one were haemorrhagic strokes. Despite the statistically significant associations with several stroke risk factors (Table 2), we did not find statistically significant associations between the estimated D5D or D6D activities and risk of incident stroke (Table 3). Entering the estimated desaturase activities as tertiles or continuously (per $1 \mathrm{sD}$ ) instead of quartiles into the models did not reveal any statistically significant associations either. For example, the hazard ratios (HR) for any stroke, ischaemic stroke and haemorrhagic stroke for $1 \mathrm{sD}$ change in the estimated D5D activity were 0.99 (95\% CI 0.86, 1.15), 0.98 (95\% CI 0.83, 1.16) and 0.95 (95\% CI 0.72, 1.27), respectively. The respective HR for the estimated D6D activity were 1.06 (95\% CI $0.93,1.22$ ), $1.09(95 \%$ CI $0.93,1.27)$ and 0.98 (95\% CI $0.75,1.29)$ (other data not shown).

Because a long follow-up could potentially attenuate the associations between exposures that are measured only at baseline, we also investigated the associations with a shorter, 12.8-year mean follow-up. However, we did not find statistically significant associations with this shorter follow-up either (multivariable-adjusted extreme-quartile $\mathrm{HR}$ for total stroke (104 events) $1.34 ; 95 \%$ CI $0.77,2.34 ; P_{\text {trend }} 0.30$ for the estimated D6D activity and HR 0.90; 95\% CI 0.50, 1.63; $P_{\text {trend }} 0.64$ for the estimated D5D activity). Because diabetes can affect the desaturase activities and many risk factors shown in Table 2, we also investigated the associations after excluding those with type 2 diabetes ( $n$ 83). However, the associations with risk factors and with risk of incident stroke remained similar (data not shown). Similarly, excluding participants on lipid-lowering or anti-inflammatory medication ( $n$ 89) had no appreciable impact on the associations (data not shown).

\section{Discussion}

In this prospective cohort study among men from eastern Finland, higher estimated D5D activity had a favourable association and higher estimated D6D activity an unfavourable association with several stroke risk factors. However, neither was associated with the risk of incident stroke.

Overall, the impact of PUFA metabolism on stroke risk is not well established. Although estimated desaturase activities have been associated with several stroke risk factors, the impact on stroke incidence has so far been scarcely investigated. Our findings of the associations between estimated D5D or D6D activities and stroke risk factors are consistent with the results from most previous studies, where high estimated D6D activity has generally had adverse associations with stroke risk factors, while high estimated D5D activity has been favourably associated. For example, higher estimated D6D activity has been associated with higher serum $\mathrm{TAG}^{(9,12,15,17)}$, higher LDLcholesterol $^{(9,15)}$, lower HDL-cholesterol ${ }^{(12,15)}$, higher $\mathrm{CRP}^{(12,14)}$, higher blood pressure ${ }^{(17)}$, higher BMI and obesity ${ }^{(10-12,15,17)}$ and higher HOMA insulin resistance ${ }^{(17)}$, whereas higher estimated D5D activity has been associated with lower serum $\operatorname{TAG}^{(8,9,12,15,17)}$, lower LDL-cholesterol ${ }^{(9)}$, higher HDLcholesterol $^{(12)}$, lower $\mathrm{CRP}^{(12,14)}$, lower blood pressure ${ }^{(8,19)}$, lower $\mathrm{BMI}^{(10-12,16)}$ and lower HOMA insulin resistance ${ }^{(17)}$ '. Although these studies have been cross-sectional, a longitudinal study also found similar differences between the estimated D5D and D6D activities ${ }^{(13)}$. Similarly, FADS1/FADS2 polymorphisms have been shown to associate, for example, with serum lipid levels and glucose metabolism ${ }^{(3,29)}$, adding more evidence for the impact of D5D and D6D activities on these risk factors. A recent study also found that two FADS1/FADS2 SNP were associated with higher risk of ischaemic stroke in a Chinese population, possibly by influencing serum lipid levels ${ }^{(30)}$.

The reasons for the opposite associations of D6D and D5D activities are not completely known. D6D activity is downregulated and $\mathrm{D} 5 \mathrm{D}$ activity up-regulated in the presence of a high-PUFA diet ${ }^{(31)}$, so the desaturase activities may reflect the beneficial effects of the essential fatty acids, especially LA, on the risk factors ${ }^{(32)}$. On the other hand, D5D is the key enzyme for production of the $n-6$ PUFA arachidonic acid and the $n-3$ PUFA EPA and DHA, which have important roles as precursors in the production of eicosanoids and other bioactive compounds ${ }^{(33)}$

Previous studies have indicated an association between estimated D6D and D5D activities and risk of CVD ${ }^{(3,34)}$. However, despite the observed associations with the stroke risk factors, estimated D5D or D6D activities were not associated with the risk of stroke in our study. This finding is consistent with the result of the only previous prospective study, which 
Table 1. Baseline characteristics according to quartiles (Q) of estimated serum $\Delta-5$-desaturase and $\Delta-6$-desaturase activities ${ }^{*}$ (Mean values and standard deviations; percentages)

\begin{tabular}{|c|c|c|c|c|c|c|c|c|c|c|}
\hline & \multicolumn{4}{|c|}{$\Delta-5$-desaturase activity } & \multirow[b]{3}{*}{$P_{\text {trend }}$} & \multicolumn{4}{|c|}{$\Delta$-6-desaturase activity } & \multirow[b]{3}{*}{$P_{\text {trend }}$} \\
\hline & \multicolumn{2}{|c|}{ Q1 (<2.99) } & \multicolumn{2}{|c|}{ Q4 (>4.28) } & & \multicolumn{2}{|c|}{ Q1 } & \multicolumn{2}{|c|}{ Q4 } & \\
\hline & Mean & SD & Mean & SD & & Mean & SD & Mean & SD & \\
\hline Number of subjects & \multicolumn{2}{|c|}{460} & \multicolumn{2}{|c|}{461} & & \multicolumn{2}{|c|}{460} & \multicolumn{2}{|c|}{460} & \\
\hline Age (years) & 52.5 & $5 \cdot 1$ & $52 \cdot 3$ & 5.6 & 0.72 & 52.8 & $5 \cdot 4$ & $51 \cdot 7$ & 5.4 & 0.003 \\
\hline Education (years) & 8.9 & 3.4 & $9 \cdot 1$ & $3 \cdot 7$ & 0.83 & $9 \cdot 1$ & 3.6 & 8.5 & 3.0 & 0.01 \\
\hline Leisure-time physical activity $(\mathrm{kJ} / \mathrm{d})$ & 540 & 732 & 623 & 653 & 0.25 & 619 & 686 & 544 & 720 & 0.30 \\
\hline$(\mathrm{kcal} / \mathrm{d})$ & 129 & 175 & 149 & 156 & & 148 & 164 & 130 & 172 & \\
\hline BMI $\left(\mathrm{kg} / \mathrm{m}^{2}\right)$ & 27.3 & 3.6 & $26 \cdot 2$ & 3.1 & $<0.001$ & 26.0 & 3.1 & $27 \cdot 4$ & 3.6 & $<0.001$ \\
\hline Marital status, married (\%) & \multicolumn{2}{|c|}{87} & \multicolumn{2}{|c|}{86} & 0.89 & \multicolumn{2}{|c|}{88} & \multicolumn{2}{|c|}{86} & 0.66 \\
\hline Living in rural area (\%) & \multicolumn{2}{|c|}{32} & \multicolumn{2}{|c|}{27} & 0.12 & \multicolumn{2}{|c|}{24} & \multicolumn{2}{|c|}{35} & $<0.001$ \\
\hline Current smoker (\%) & \multicolumn{2}{|c|}{29} & \multicolumn{2}{|c|}{28} & 0.59 & \multicolumn{2}{|c|}{31} & \multicolumn{2}{|c|}{27} & 0.27 \\
\hline Diabetes (\%) & \multirow{2}{*}{\multicolumn{2}{|c|}{$\begin{array}{c}5 \\
19\end{array}$}} & \multirow{2}{*}{\multicolumn{2}{|c|}{$\begin{array}{c}4 \\
19\end{array}$}} & 0.55 & \multicolumn{2}{|c|}{4} & \multicolumn{2}{|c|}{6} & 0.04 \\
\hline Family history of stroke (\%) & & & & & 0.96 & \multirow{2}{*}{\multicolumn{2}{|c|}{$\begin{array}{l}19 \\
68\end{array}$}} & & & 0.50 \\
\hline Hypertension medication before stroke (\%) & \multicolumn{2}{|c|}{72} & \multicolumn{2}{|c|}{69} & 0.52 & & & & & 0.14 \\
\hline Alcohol intake (g/week) & 59 & 105 & 98 & 139 & $<0.001$ & 59 & 94 & 96 & 158 & $<0.001$ \\
\hline Total serum $n-3$ PUFA (\%) & 4.6 & 1.0 & 6.5 & $2 \cdot 0$ & $<0.001$ & 5.4 & 1.6 & $5 \cdot 2$ & 1.3 & 0.01 \\
\hline$a$-Linolenic acid (\%) & 0.8 & 0.3 & 0.7 & 0.2 & $<0.001$ & 0.8 & 0.2 & 0.7 & 0.2 & $<0.001$ \\
\hline EPA (\%) & 1.2 & 0.5 & $2 \cdot 2$ & 1.3 & $<0.001$ & 1.6 & 0.9 & 1.7 & 0.7 & 0.08 \\
\hline DPA (\%) & 0.5 & 1.0 & 0.6 & $0 \cdot 1$ & $<0.001$ & 0.5 & 0.1 & 0.6 & 0.1 & 0.001 \\
\hline DHA (\%) & 2.0 & 0.5 & 3.0 & 0.8 & $<0.001$ & 2.6 & 0.7 & 2.3 & 0.7 & $<0.001$ \\
\hline Total serum $n-6$ PUFA (\%) & $29 \cdot 7$ & 4.5 & 33.2 & 4.4 & $<0.001$ & $34 \cdot 3$ & $4 \cdot 2$ & 28.6 & $4 \cdot 3$ & $<0.001$ \\
\hline$\gamma$-Linolenic acid (\%) & 0.3 & 0.1 & 0.2 & $0 \cdot 1$ & $<0.001$ & 0.2 & 0.04 & 0.4 & 0.1 & $<0.001$ \\
\hline Dihomo- $\gamma$-linolenic acid (\%) & 1.5 & 0.2 & 1.1 & 0.2 & $<0.001$ & 1.2 & 0.2 & 1.5 & 0.2 & $<0.001$ \\
\hline Linoleic acid (\%) & 25.4 & $4 \cdot 3$ & $27 \cdot 3$ & 4.5 & $<0.001$ & 29.5 & 4.0 & 23.3 & 3.8 & $<0.001$ \\
\hline Arachidonic acid (\%) & 3.9 & 0.7 & 5.7 & 1.0 & $<0.001$ & 4.6 & 1.0 & 2.9 & $1 \cdot 0$ & $<0.001$ \\
\hline
\end{tabular}

${ }^{*} \Delta-5$-desaturase activity was calculated as the ratio of arachidonic acid:dihomo- $\gamma$-linolenic acid and $\Delta-6$-desaturase activity as the ratio of $\gamma$-linolenic acid:linoleic acid. 


\section{NS British Journal of Nutrition}

Table 2. Associations of the estimated serum $\Delta$-5-desaturase and $\Delta$-6-desaturase activities with risk factors for stroke in 1842 men from the Kuopio Ischaemic Heart Disease Risk Factor Study (Mean values with their standard errors)

\begin{tabular}{|c|c|c|c|c|c|c|c|c|c|}
\hline & \multicolumn{8}{|c|}{ Quartile of serum desaturase activity } & \multirow[b]{3}{*}{$P_{\text {trend }}$} \\
\hline & \multicolumn{2}{|c|}{ Q1 } & \multicolumn{2}{|c|}{ Q2 } & \multicolumn{2}{|c|}{ Q3 } & \multicolumn{2}{|c|}{ Q4 } & \\
\hline & Mean & SEM & Mean & SEM & Mean & SEM & Mean & SEM & \\
\hline \multicolumn{10}{|c|}{ Systolic blood pressure $(\mathrm{mmHg})$} \\
\hline$\Delta-5$ desaturase & \multicolumn{2}{|c|}{$<2.99$} & \multicolumn{2}{|c|}{$2.99-3.51$} & \multicolumn{2}{|c|}{$3.52-4.28$} & \multicolumn{2}{|c|}{$>4.28$} & \\
\hline Model 1† & 136 & $0 \cdot 8$ & 134 & $0 \cdot 8$ & 134 & 0.8 & 132 & 0.8 & 0.002 \\
\hline Model 2ł & 135 & 0.7 & 134 & 0.7 & 134 & 0.7 & 133 & 0.7 & 0.04 \\
\hline$\Delta-6$ desaturase & & & & & & & & & \\
\hline Model 1 & 132 & 0.8 & 134 & 0.8 & 134 & 0.8 & 137 & 0.8 & $<0.001$ \\
\hline Model 2 & 133 & 0.7 & 135 & 0.7 & 134 & 0.7 & 136 & 0.7 & 0.02 \\
\hline \multicolumn{10}{|c|}{ Diastolic blood pressure $(\mathrm{mmHg})$} \\
\hline \multicolumn{10}{|l|}{$\Delta-5$ desaturase } \\
\hline Model 1 & 90 & 0.5 & 89 & 0.5 & 89 & 0.5 & 88 & 0.5 & $<0.001$ \\
\hline Model 2 & 90 & 0.5 & 89 & 0.5 & 89 & 0.4 & 88 & 0.5 & 0.005 \\
\hline \multicolumn{10}{|l|}{$\Delta-6$ desaturase } \\
\hline Model 1 & 87 & 0.5 & 89 & 0.5 & 89 & 0.5 & 91 & 0.5 & $<0.001$ \\
\hline Model 2 & 88 & 0.5 & 89 & 0.5 & 89 & 0.4 & 90 & 0.5 & 0.02 \\
\hline \multicolumn{10}{|l|}{ BMI $\left(\mathrm{kg} / \mathrm{m}^{2}\right)$} \\
\hline \multicolumn{10}{|l|}{$\Delta-5$ desaturase } \\
\hline Model 1 & $27 \cdot 3$ & $0 \cdot 2$ & $26 \cdot 8$ & 0.2 & $26 \cdot 5$ & 0.2 & $26 \cdot 2$ & 0.2 & $<0.001$ \\
\hline Model 2 & $27 \cdot 4$ & 0.2 & $26 \cdot 9$ & 0.2 & $26 \cdot 5$ & 0.2 & $26 \cdot 1$ & 0.2 & $<0.001$ \\
\hline \multicolumn{10}{|l|}{$\Delta-6$ desaturase } \\
\hline Model 1 & $26 \cdot 0$ & 0.2 & $26 \cdot 5$ & 0.2 & $26 \cdot 9$ & 0.2 & 27.5 & 0.2 & $<0.001$ \\
\hline Model 2 & $26 \cdot 0$ & 0.2 & $26 \cdot 5$ & 0.2 & $26 \cdot 9$ & 0.2 & $27 \cdot 4$ & 0.2 & $<0.001$ \\
\hline \multicolumn{10}{|c|}{ LDL-cholesterol (mmol/l) } \\
\hline \multicolumn{10}{|l|}{$\Delta-5$ desaturase } \\
\hline Model 1 & 3.9 & 0.04 & 4.0 & 0.1 & 4.0 & 0.04 & 4.0 & 0.04 & 0.11 \\
\hline Model 2 & 3.9 & 0.04 & 4.0 & 0.04 & $4 \cdot 1$ & 0.04 & 4.0 & 0.1 & 0.09 \\
\hline \multicolumn{10}{|l|}{$\Delta-6$ desaturase } \\
\hline Model 1 & 3.9 & 0.04 & 4.0 & 0.04 & 4.1 & 0.04 & 4.0 & 0.04 & 0.17 \\
\hline Model 2 & 3.9 & $0 \cdot 1$ & 4.0 & 0.04 & $4 \cdot 1$ & 0.04 & 4.0 & $0 \cdot 1$ & 0.15 \\
\hline HDL-cholesterol ( $r$ & & & & & & & & & \\
\hline$\Delta-5$ desaturase & & & & & & & & & \\
\hline Model 1 & $1 \cdot 2$ & 0.01 & 1.3 & 0.01 & 1.3 & 0.01 & 1.4 & 0.01 & $<0.001$ \\
\hline Model 2 & 1.2 & 0.01 & 1.3 & 0.01 & 1.3 & 0.01 & 1.4 & 0.01 & $<0.001$ \\
\hline$\Delta-6$ desaturase & & & & & & & & & \\
\hline Model 1 & 1.3 & 0.01 & 1.3 & 0.01 & 1.3 & 0.01 & 1.3 & 0.01 & 0.21 \\
\hline Model 2 & 1.3 & 0.01 & 1.3 & 0.01 & 1.3 & 0.01 & 1.3 & 0.01 & 0.66 \\
\hline TAG $(\mathrm{mmol} / \mathrm{l})$ & & & & & & & & & \\
\hline$\Delta-5$ desaturase & & & & & & & & & \\
\hline Model 1 & 1.6 & 0.03 & 1.4 & 0.03 & 1.1 & 0.03 & 1.0 & 0.03 & $<0.001$ \\
\hline Model 2 & 1.6 & 0.03 & 1.4 & 0.03 & $1 \cdot 1$ & 0.03 & 1.0 & 0.03 & $<0.001$ \\
\hline$\Delta-6$ desaturase & & & & & & & & & \\
\hline Model 1 & 1.1 & 0.03 & 1.2 & 0.03 & 1.3 & 0.03 & 1.5 & 0.03 & $<0.001$ \\
\hline Model 2 & 1.1 & 0.03 & 1.2 & 0.03 & 1.3 & 0.03 & 1.5 & 0.03 & $<0.001$ \\
\hline Blood glucose (mr & & & & & & & & & \\
\hline$\Delta-5$ desaturase & & & & & & & & & \\
\hline Model 1 & 4.7 & 0.04 & 4.7 & 0.04 & 4.7 & 0.04 & 4.7 & 0.04 & 0.20 \\
\hline Model 2 & 4.7 & 0.04 & 4.7 & 0.04 & 4.7 & 0.04 & 4.7 & 0.04 & 0.68 \\
\hline
\end{tabular}


Nesitish Journal of Nutrition

\begin{tabular}{|c|c|c|c|c|c|c|c|c|c|}
\hline & \multicolumn{8}{|c|}{ Quartile of serum desaturase activity } & \multirow[b]{3}{*}{$P_{\text {trend }}$} \\
\hline & \multicolumn{2}{|c|}{ Q1 } & \multicolumn{2}{|c|}{ Q2 } & \multicolumn{2}{|c|}{ Q3 } & \multicolumn{2}{|c|}{ Q4 } & \\
\hline & Mean & SEM & Mean & SEM & Mean & SEM & Mean & SEM & \\
\hline \multicolumn{10}{|c|}{$\Delta-6$ desaturase } \\
\hline Model 1 & 4.6 & 0.04 & 4.7 & 0.04 & 4.7 & 0.04 & 4.8 & 0.04 & 0.01 \\
\hline Model 2 & 4.7 & 0.04 & 4.7 & 0.04 & 4.7 & 0.04 & 4.7 & 0.04 & 0.30 \\
\hline \multicolumn{10}{|c|}{ Serum insulin (mU/l) } \\
\hline \multicolumn{10}{|c|}{$\Delta-5$ desaturase } \\
\hline Model 1 & $13 \cdot 1$ & 0.3 & 11.3 & 0.3 & $10 \cdot 4$ & 0.3 & $9 \cdot 6$ & 0.3 & $<0.001$ \\
\hline Model 2 & $12 \cdot 4$ & 0.3 & 11.2 & 0.3 & $10 \cdot 6$ & 0.3 & $10 \cdot 2$ & 0.3 & $<0.001$ \\
\hline \multicolumn{10}{|c|}{$\Delta-6$ desaturase } \\
\hline Model 1 & 9.9 & 0.3 & $10 \cdot 6$ & 0.3 & 11.6 & 0.3 & 12.4 & 0.3 & $<0.001$ \\
\hline Model 2 & $10 \cdot 6$ & 0.3 & $10 \cdot 8$ & 0.3 & 11.4 & 0.3 & $11 \cdot 6$ & 0.3 & 0.01 \\
\hline \multicolumn{10}{|c|}{ C-reactive protein (mg/l) } \\
\hline \multicolumn{10}{|c|}{$\Delta-5$ desaturase } \\
\hline Model 1 & 2.8 & 0.2 & $2 \cdot 1$ & 0.2 & $2 \cdot 1$ & 0.2 & $2 \cdot 0$ & 0.2 & 0.01 \\
\hline Model 2 & $2 \cdot 7$ & 0.2 & $2 \cdot 1$ & 0.2 & $2 \cdot 2$ & 0.2 & 1.9 & 0.2 & 0.01 \\
\hline \multicolumn{10}{|c|}{$\Delta-6$ desaturase } \\
\hline Model 1 & 2.0 & 0.2 & $2 \cdot 2$ & 0.2 & 2.4 & 0.2 & $2 \cdot 4$ & 0.2 & $0 \cdot 16$ \\
\hline Model 2 & $2 \cdot 1$ & 0.2 & $2 \cdot 2$ & 0.2 & 2.4 & 0.2 & $2 \cdot 3$ & 0.2 & 0.44 \\
\hline \multicolumn{10}{|c|}{ HOMA insulin resistance } \\
\hline \multicolumn{10}{|c|}{$\Delta-5$ desaturase } \\
\hline Model 1 & 1.6 & 0.04 & 1.5 & 0.04 & 1.3 & 0.04 & 1.3 & 0.04 & $<0.001$ \\
\hline Model 2 & 1.6 & 0.03 & 1.5 & 0.03 & 1.4 & 0.03 & 1.3 & 0.03 & $<0.001$ \\
\hline \multicolumn{10}{|c|}{$\Delta-6$ desaturase } \\
\hline Model 1 & 1.3 & 0.04 & 1.4 & 0.04 & 1.5 & 0.04 & 1.6 & 0.04 & $<0.001$ \\
\hline Model 2 & 1.4 & 0.03 & 1.4 & 0.03 & 1.5 & 0.03 & 1.5 & 0.03 & $<0.001$ \\
\hline \multirow{2}{*}{\multicolumn{10}{|c|}{ HOMA $\beta$-cell function }} \\
\hline & & & & & & & & & \\
\hline Model 1 & $118 \cdot 6$ & 1.7 & $112 \cdot 8$ & 1.8 & $106 \cdot 7$ & 1.7 & $103 \cdot 2$ & 1.7 & $<0.001$ \\
\hline Model 2 & $117 \cdot 1$ & 1.6 & 114.1 & 1.6 & $109 \cdot 7$ & 1.6 & 107.5 & 1.6 & $<0.001$ \\
\hline \multicolumn{10}{|c|}{$\Delta-6$ desaturase } \\
\hline Model 1 & 105.9 & 1.8 & $107 \cdot 2$ & 1.8 & $113 \cdot 2$ & 1.8 & 114.9 & 1.8 & $<0.001$ \\
\hline Model 2 & $108 \cdot 3$ & 1.7 & 107.9 & 1.7 & $112 \cdot 3$ & 1.7 & $112 \cdot 7$ & 1.7 & 0.03 \\
\hline \multicolumn{10}{|c|}{ HOMA insulin sensitivity } \\
\hline \multicolumn{10}{|c|}{$\Delta-5$ desaturase } \\
\hline Model 1 & $75 \cdot 2$ & 1.7 & $81 \cdot 3$ & 1.7 & 91.0 & 1.7 & $95 \cdot 2$ & 1.7 & $<0.001$ \\
\hline Model 2 & 78.4 & 1.5 & $82 \cdot 0$ & 1.5 & 90.0 & 1.5 & $92 \cdot 4$ & 1.5 & $<0.001$ \\
\hline \multicolumn{10}{|c|}{$\Delta-6$ desaturase } \\
\hline Model 1 & $95 \cdot 2$ & 1.7 & $89 \cdot 7$ & 1.7 & 81.9 & 1.7 & $76 \cdot 3$ & 1.7 & $<0.001$ \\
\hline Model 2 & 91.3 & 1.5 & 88.5 & 1.5 & 83.1 & 1.5 & $80 \cdot 1$ & 1.6 & $<0.001$ \\
\hline
\end{tabular}

Q, quartile; HOMA, homoeostasis model assessment

${ }^{*} \Delta-5$-desaturase activity was calculated as the ratio of arachidonic acid:dihomo- - -linolenic acidy and $\Delta-6$-desaturase activity as the ratio of $\gamma$-linolenic acid:linoleic acid.

† Model 2: adjusted for model 1 plus BMI, smoking, physical activity and alcohol intake. 
Table 3. Risk of incident total stroke, ischaemic stroke and haemorrhagic stroke in quartiles (Q) of estimated serum $\Delta-5$-desaturase and $\Delta-6$-desaturase indices ${ }^{*}$ (Hazard ratios (HR) and $95 \%$ confidence intervals)

\begin{tabular}{|c|c|c|c|c|c|c|c|c|c|}
\hline & \multicolumn{8}{|c|}{ Quartile of serum desaturase activities } & \multirow[b]{3}{*}{$P_{\text {trend }}$} \\
\hline & \multicolumn{2}{|c|}{ Q1 } & \multicolumn{2}{|c|}{ Q2 } & \multicolumn{2}{|c|}{ Q3 } & \multicolumn{2}{|c|}{ Q4 } & \\
\hline & $\mathrm{HR}$ & $95 \% \mathrm{Cl}$ & $\mathrm{HR}$ & $95 \% \mathrm{Cl}$ & $\mathrm{HR}$ & $95 \% \mathrm{Cl}$ & $\mathrm{HR}$ & $95 \% \mathrm{Cl}$ & \\
\hline \multicolumn{10}{|c|}{ All stroke cases ( $n$ 202) } \\
\hline$\Delta-5$ desaturase & \multicolumn{2}{|c|}{$\begin{array}{l}<2.99 \\
(n 460)\end{array}$} & \multicolumn{2}{|c|}{$\begin{array}{l}2 \cdot 99-3.51 \\
(n 461)\end{array}$} & \multicolumn{2}{|c|}{$\begin{array}{l}3 \cdot 51-4 \cdot 28 \\
(n 461)\end{array}$} & \multicolumn{2}{|c|}{$\begin{array}{l}>4.28 \\
(n 460)\end{array}$} & \\
\hline Events $(n)$ & \multicolumn{2}{|c|}{47} & \multicolumn{2}{|c|}{61} & \multicolumn{2}{|c|}{47} & \multicolumn{2}{|c|}{47} & \\
\hline Model $1 \dagger$ & 1 & Ref. & 1.29 & $0.88,1.88$ & 0.95 & $0.63,1.42$ & 0.98 & $0.65,1.46$ & 0.54 \\
\hline Model $2 \ddagger$ & 1 & Ref. & 1.35 & $0.92,1.98$ & 1.01 & $0.67,1.51$ & 1.06 & $0.70,1.60$ & 0.81 \\
\hline$\Delta-6$ desaturase & \multirow{2}{*}{\multicolumn{2}{|c|}{$\begin{array}{c}<0.007 \\
(n 460) \\
50\end{array}$}} & \multicolumn{2}{|c|}{$\begin{array}{l}0.007-0.010 \\
\quad(n \text { 456) }\end{array}$} & \multicolumn{2}{|c|}{$\begin{array}{c}0.011-0.014 \\
(n \text { 465) }\end{array}$} & \multicolumn{2}{|c|}{$\begin{array}{l}>0.014 \\
(n \text { 461) }\end{array}$} & \\
\hline Events $(n)$ & & & \multicolumn{2}{|c|}{44} & \multicolumn{2}{|c|}{$\begin{array}{c}(n 465) \\
51\end{array}$} & \multicolumn{2}{|c|}{$\begin{array}{c}(n+6) 1) \\
57\end{array}$} & \\
\hline Model 1 & 1 & Ref. & 0.89 & $0.59,1.33$ & 1.07 & $0.73,1.59$ & 1.27 & $0.87,1.85$ & 0.12 \\
\hline Model 2 & 1 & Ref. & 0.87 & $0.58,1.31$ & 1.02 & $0.69,1.51$ & $1 \cdot 18$ & $0.80,1.74$ & 0.25 \\
\hline \multicolumn{10}{|c|}{ Ischaemic stroke cases ( $n$ 153) } \\
\hline \multicolumn{10}{|c|}{$\Delta-5$ desaturase } \\
\hline Events $(n)$ & \multicolumn{2}{|c|}{37} & \multicolumn{2}{|c|}{50} & \multicolumn{2}{|c|}{32} & \multicolumn{2}{|c|}{34} & \\
\hline Model 1 & 1 & Ref. & 1.33 & $0.87,2.03$ & 0.81 & $0.51,1.30$ & 0.89 & $0.56,1.42$ & 0.27 \\
\hline Model 2 & 1 & Ref. & 1.41 & $0.92,2 \cdot 16$ & 0.88 & $0.55,1.41$ & 1.00 & $0.62,1.60$ & 0.54 \\
\hline \multicolumn{10}{|l|}{$\Delta-6$ desaturase } \\
\hline Events $(n)$ & & & & & & & & & \\
\hline Model 1 & 1 & Ref. & 0.96 & $0.61,1.51$ & 0.95 & $0.60,1.51$ & 1.31 & $0.85,2.03$ & 0.17 \\
\hline Model 2 & 1 & Ref. & 0.94 & $0.59,1.48$ & 0.90 & $0.57,1.44$ & 1.24 & $0.80,1.93$ & 0.27 \\
\hline Haemorrhagic str & 75) & & & & & & & & \\
\hline$\Delta-5$ desaturase & & & & & & & & & \\
\hline Events $(n)$ & & & & & & & & & \\
\hline Model 1 & 1 & Ref. & $1 \cdot 20$ & $0.54,2.67$ & 1.39 & $0.65,3.00$ & 1.00 & $0.43,2.31$ & 0.99 \\
\hline Model 2 & 1 & Ref. & 1.24 & $0.55,2.76$ & 1.48 & $0.68,3.20$ & 1.03 & $0.44,2.43$ & 0.94 \\
\hline$\Delta-6$ desaturase & & & & & & & & & \\
\hline Events $(n)$ & & & & & & & & & \\
\hline Model 1 & 1 & Ref. & 0.68 & $0.28,1.68$ & 1.49 & $0.71,3.11$ & $1 \cdot 24$ & $0.57,2.69$ & 0.32 \\
\hline Model 2 & 1 & Ref. & 0.66 & $0.27,1.63$ & 1.39 & $0.66,2.92$ & 1.06 & $0.48,2.33$ & 0.58 \\
\hline
\end{tabular}

Ref., referent values.

* $\Delta$-5-desaturase activity was calculated as the ratio of arachidonic acid:dihomo- $\gamma$-linolenic acidy and $\Delta$-6-desaturase activity as the ratio of $\gamma$-linolenic acid:linoleic acid.

1: adjusted for age and examination year.

T Model 2: adjusted for model 1 plus BMI, smoking, physical activity and alcohol intake. 
did not find statistically significant associations between estimated D5D or D6D activities and risk of stroke ${ }^{(7)}$. A possible explanation for why we found an association with stroke risk factors but not with stroke incidence may be that the impact of the desaturase enzymes on the risk factors is too small and weak to have a significant impact on the risk of stroke. Especially the association with one of the strongest risk factors for stroke, high blood pressure, was quite modest and may not be clinically significant. The lack of association with the desaturase activities in the current study is also supported by the similar lack of association of the serum $n-3$ and $n-6$ PUFA with the risk of stroke in this study population ${ }^{(28)}$

The strengths of the present study include the prospective and population-based design, and the extensive database of potential confounders and mediators. Several limitations should also be acknowledged. First, a potential weakness is the use of fatty acid product:precursor ratio to estimate hepatic desaturase activities indirectly, instead of directly measuring the desaturase activity. Direct measurement of enzyme activity is very impractical in large studies, but the use of a product:precursor ratio as a substitute measure to estimate desaturase activity is well established ${ }^{(3,20,31)}$. However, desaturase activities are commonly estimated from phospholipid or cholesterol ester fatty acids, not from the whole serum fatty acids like in our study. This makes direct comparison of the estimated desaturase activities challenging. For example, because the proportion of $\gamma$-linolenic acid in phospholipids is usually very low, the estimated D6D activity when using phospholipid fatty acids is calculated as the ratio of dihomo- $\gamma$-linolenic acid:LA, which also describes the activity of the elongase that converts $\gamma$-linolenic acid to dihomo- $\gamma$-linolenic acid. However, D5D and D6D activities estimated from whole serum have been shown to strongly associate with a known intron variant of the FADS1 gene, which provides indirect validation for the use of whole serum fatty acids as well to estimate the desaturase activities ${ }^{(35)}$. Second, we had information on the estimated desaturase activities only from the baseline, which may not be representative of the entire long follow-up. This could potentially create exposure misclassification and thus random error, which would attenuate the associations towards the null in analyses with incident stroke events. Because this kind of error would not affect the cross-sectional analyses with the risk factors, the discrepancy between the findings with risk factors and with the risk of incident events may at least partly be explained by the lack of repeated measurement of desaturase activities. On the other hand, the cross-sectional analyses are prone to reverse causation. Third, because of the low number of especially haemorrhagic stroke events, the findings regarding the associations between the desaturase activities and stroke risk should be interpreted cautiously. However, we did not find any associations with stroke risk when we analysed the associations in tertiles or continuously instead of using quartiles, which indicates that the lack of association with incident events may not be due to the low number of events. Finally, the study included only middle-aged and older Caucasian men, so the results may not be generalisable to other populations or to women.

In summary, estimated D5D and D6D activities were both associated with several risk factors for stroke: higher estimated
D5D activity showing generally favourable associations and higher estimated D6D activity unfavourable associations. In spite of these associations with the risk factors, the desaturase activities were not associated with risk of incident stroke. Further studies in larger study populations are needed to elucidate the impact of D5D and D6D activities on the risk of stroke. In addition, there is a need for more studies to clarify what factors determine the activities of these enzymes and what biological mechanisms could explain the opposite associations of these desaturases with several risk factors for chronic diseases.

\section{Acknowledgements}

The study was supported by a grant from Juho Vainio Foundation to R. D. The funding body had no role in study design, in the collection, analysis and interpretation of data, in the writing of the report or in the decision to submit the article for publication.

The authors' contributions were as follows: R. D., S. K., T.-P. T. and J. K. V. contributed to the conception and design of the research; S. K. and T.-P. T. acquired data; R. D. and J. K. V. analysed data and interpreted the results; R. D. drafted the manuscript. All authors critically revised the paper and approved the final version.

None of the authors has any conflicts of interest to declare.

\section{References}

1. Mozaffarian D \& Wu JH (2011) Omega-3 fatty acids and cardiovascular disease: effects on risk factors, molecular pathways, and clinical events. J Am Coll Cardiol 58, 2047-2067.

2. Harris WS, Mozaffarian D, Rimm E, et al. (2009) Omega-6 fatty acids and risk for cardiovascular disease: a science advisory from the American heart association nutrition subcommittee of the council on nutrition, physical activity, and metabolism; council on cardiovascular nursing; and council on epidemiology and prevention. Circulation 119, 902-907.

3. Tosi F, Sartori F, Guarini P, et al. (2014) Delta-5 and delta-6 desaturases: crucial enzymes in polyunsaturated fatty acidrelated pathways with pleiotropic influences in health and disease. Adv Exp Med Biol 824, 61-81.

4. Cho HP, Nakamura MT \& Clarke SD (1999) Cloning, expression, and nutritional regulation of the mammalian delta-6 desaturase. J Biol Chem 274, 471-477.

5. Cho HP, Nakamura M \& Clarke SD (1999) Cloning, expression, and fatty acid regulation of the human delta-5 desaturase. J Biol Chem 274, 37335-37339.

6. Lemaitre RN, Tanaka T, Tang W, et al. (2011) Genetic loci associated with plasma phospholipid $n-3$ fatty acids: a metaanalysis of genome-wide association studies from the CHARGE Consortium. PLoS Genet 7, e1002193.

7. Yamagishi K, Folsom AR, Steffen LM, et al. (2013) Plasma fatty acid composition and incident ischemic stroke in middle-aged adults: the Atherosclerosis Risk in Communities (ARIC) Study. Cerebrovasc Dis 36, 38-46.

8. Kim SR, Jeon SY \& Lee SM (2015) The association of cardiovascular risk factors with saturated fatty acids and fatty acid desaturase indices in erythrocyte in middle-aged Korean adults. Lipids Health Dis 14, 133

9. Jacobs S, Schiller K, Jansen E, et al. (2014) Association between erythrocyte membrane fatty acids and biomarkers of dyslipidemia in the EPIC-Potsdam study. Eur J Clin Nutr 68, $517-525$. 
10. Warensio E, Rosell M, Hellenius ML, et al. (2009) Associations between estimated fatty acid desaturase activities in serum lipids and adipose tissue in humans: links to obesity and insulin resistance. Lipids Health Dis $\mathbf{8}, 37$.

11. Warensjo E, Riserus U \& Vessby B (2005) Fatty acid composition of serum lipids predicts the development of the metabolic syndrome in men. Diabetologia 48, 1999-2005.

12. Do HJ, Chung HK, Moon J, et al. (2011) Relationship between the estimates of desaturase activities and cardiometabolic phenotypes in Koreans. J Clin Biochem Nutr 49, 131-135.

13. Petersson H, Basu S, Cederholm T, et al. (2008) Serum fatty acid composition and indices of stearoyl-CoA desaturase activity are associated with systemic inflammation: longitudinal analyses in middle-aged men. Br J Nutr 99, 1186-1189.

14. Poudel-Tandukar K, Sato M, Ejima Y, et al. (2012) Relationship of serum fatty acid composition and desaturase activity to C-reactive protein in Japanese men and women. Atherosclerosis 220, 520-524.

15. Steffen LM, Vessby B, Jacobs DR Jr, et al. (2008) Serum phospholipid and cholesteryl ester fatty acids and estimated desaturase activities are related to overweight and cardiovascular risk factors in adolescents. Int $J$ Obes (Lond) 32, $1297-1304$.

16. Kawashima A, Sugawara S, Okita M, et al. (2009) Plasma fatty acid composition, estimated desaturase activities, and intakes of energy and nutrient in Japanese men with abdominal obesity or metabolic syndrome. J Nutr Sci Vitaminol (Tokyo) 55, 400-406.

17. Murakami K, Sasaki S, Takahashi Y, et al. (2008) Lower estimates of delta- 5 desaturase and elongase activity are related to adverse profiles for several metabolic risk factors in young Japanese women. Nutr Res 28, 816-824.

18. Salonen JT (1988) Is there a continuing need for longitudinal epidemiologic research? The Kuopio Ischaemic Heart Disease Risk Factor Study. Ann Clin Res 20, 46-50.

19. Laaksonen DE, Lakka TA, Lakka HM, et al. (2002) Serum fatty acid composition predicts development of impaired fasting glycaemia and diabetes in middle-aged men. Diabet Med 19, 456-464.

20. Hodson L, Skeaff CM \& Fielding BA (2008) Fatty acid composition of adipose tissue and blood in humans and its use as a biomarker of dietary intake. Prog Lipid Res 47, 348-380.

21. Salonen JT, Nyyssonen K, Korpela H, et al. (1992) High stored iron levels are associated with excess risk of myocardial infarction in eastern Finnish men. Circulation 86, 803-811.

22. Wallace TM \& Matthews DR (2002) The assessment of insulin resistance in man. Diabet Med 19, 527-534.
23. Matthews DR, Hosker JP, Rudenski AS, et al. (1985) Homeostasis model assessment: insulin resistance and betacell function from fasting plasma glucose and insulin concentrations in man. Diabetologia 28, 412-419.

24. Lakka TA, Venalainen JM, Rauramaa R, et al. (1994) Relation of leisure-time physical activity and cardiorespiratory fitness to the risk of acute myocardial infarction. $N$ Engl J Med 330, 1549-1554.

25. Voutilainen S, Rissanen TH, Virtanen J, et al. (2001) Low dietary folate intake is associated with an excess incidence of acute coronary events: The Kuopio Ischemic Heart Disease Risk Factor Study. Circulation 103, 2674-2680.

26. Kurl S, Laukkanen JA, Rauramaa R, et al. (2003) Cardiorespiratory fitness and the risk for stroke in men. Arch Intern Med 163, 1682-1688.

27. Kurl S, Tuomainen TP, Laukkanen JA, et al. (2002) Plasma vitamin $\mathrm{C}$ modifies the association between hypertension and risk of stroke. Stroke 33, 1568-1573.

28. Daneshmand R, Kurl S, Tuomainen TP, et al. (2016) Associations of serum $n-3$ and n-6 PUFA and hair mercury with the risk of incident stroke in men: the Kuopio Ischaemic Heart Disease Risk Factor Study (KIHD). Br J Nutr 115, $1851-1859$

29. Kroger J \& Schulze MB (2012) Recent insights into the relation of delta5 desaturase and delta6 desaturase activity to the development of type 2 diabetes. Curr Opin Lipidol 23, 4-10.

30. Yang Q, Yin RX, Cao XL, et al. (2015) Association of two polymorphisms in the FADS1/FADS2 gene cluster and the risk of coronary artery disease and ischemic stroke. Int J Clin Exp Pathol 8, 7318-7331.

31. Vessby B, Gustafsson IB, Tengblad S, et al. (2002) Desaturation and elongation of fatty acids and insulin action. Ann NY Acad Sci 967, 183-195.

32. Mozaffarian D (2016) Dietary and policy priorities for cardiovascular disease, diabetes, and obesity: a comprehensive review. Circulation 133, 187-225.

33. Calder PC (2006) Polyunsaturated fatty acids and inflammation. Prostaglandins Leukot Essent Fatty Acids 75, 197-202.

34. Warensjo E, Sundstrom J, Vessby B, et al. (2008) Markers of dietary fat quality and fatty acid desaturation as predictors of total and cardiovascular mortality: a population-based prospective study. Am J Clin Nutr 88, 203-209.

35. Takkunen MJ, Schwab US, de Mello VD, et al. (2016) Longitudinal associations of serum fatty acid composition with type 2 diabetes risk and markers of insulin secretion and sensitivity in the Finnish Diabetes Prevention Study. Eur J Nutr 55, 967-979. 